\title{
FROM REALITY TO PARAMETRIC MODELS OF CULTURAL HERITAGE ASSETS FOR HBIM
}

\author{
Filippo Diara ${ }^{1 *}$, Fulvio Rinaudo ${ }^{1}$ \\ ${ }^{1}$ DAD, Department of Architecture and Design, Politecnico di Torino, Viale Mattioli 39, 10129 Torino, Italy, \\ filippo.diara@polito.it, fulvio.rinaudo@polito.it
}

KEY WORDS: Cultural Heritage, HBIM, Level of detail, NURBS, Parametric modelling, Semantics

\begin{abstract}
:
The ability of managing big amounts of metric information coming from a LiDAR survey and the ability to reproduce high quality 3D models from them are still vivid problems to solve. Is it possible to create detailed models, geometrically and metrically correct, without using a large amount (often redundant) of metric data, such as massive point clouds? Obviously yes, but there are several ways to create a fitting 3D model for a specific research. A good solution is given by NURBS based algorithms that ensure high details of modelling. However, NURBS models can’t be used directly on BIM platforms, because they need to be parametrized. In this sense, a parametric model is based on real measurements but each object could be interpreted and approximated based on objective and subjective (critic) view and also based on LODs (levels of detail or development) concerning a particular analysis. This kind of modelling of Cultural Heritage assets, fundamental for HBIM creation, need to be correctly planned especially for classification and definition of its historical features connected to an informative system, because nowadays information and then the semantic dimension are a necessary key points towards documentation analysis.

Established this brief introduction, this schematic work will focus on the analysis of FreeCAD open BIM software and Rhinoceros as NURBS 3D modeller for Cultural Heritage is concerned, and whether and how they could integrate their tools for the purpose of managing dynamic high detailed data for the creation of an HBIM platform.
\end{abstract}

\section{INTRODUCTION}

Critic and objective knowledge must be a fundamental operation to perform any kinds of analyses and researches aiming at developing design, restorative or conservative actions. This kind of knowledge, and more generally information, defines BIM (Building Information Modelling) systems.

As is nowadays it is known, BIM extends the possibilities of a traditional CAD software, allowing to set up geometric and semantic relations between 3D parametric objects and any kind of information. Indeed, one of the most important advantages of BIM solution is to combine the geometric information with internal data (related to IFC objects) and external data, such as database management systems (Diara et al., 2018).

However, BIM of existing buildings and facilities or historical buildings (and then HBIM) is still an ongoing research field and also a challenge, due to the complexity of geometric data and its interpretation inside a BIM platform (Murphy et al., 2013; Barazzetti, 2016). This issue brings the specialists to think about the need to create a fitting and custom-made solution, able to manage information and geometric features of Cultural Heritage assets, moreover, setting up an informative system of existing buildings requires some precautions, especially as far as definition and classifications of particular surfaces and work evidences, architectural components, database of materials are concerned (Beltramo et al., 2019).

As there are no standard classification or predefined protocols for HBIM creation, there are different ways and solutions to test how we can adapt custom workflow, software, formats to Cultural Heritage case study and not the opposite way of acting. The main rules to create and validate HBIM models of existing historical buildings should be the accuracy, the quality of information and the sharing process.
This work is focus on the analysis of an open source BIM software, FreeCAD, and Rhinoceros as 3D modeller software for Cultural Heritage is concerned, and whether and how they could cooperate together for the purpose to integrate free form and accurate modelling into a BIM platform.

It must be noticed that the level of accuracy of the modelling phase depends on the data that must be added to the geometric model by considering that HBIM platforms are not instruments that can be used to show detailed metric 3D as-built models.

This experimental workflow has been tested on an historical rib vault, especially the Grade of Generation and Accuracy by using NURBS modelling. In fact, starting from LiDAR data, reality-based model was created, managed and edited in order to extract curves and sections, on which were modelled NURBS surfaces and solids. Finally, these 3D objects were parametrized using customized parameters inside FreeCAD software and its add-on and additional workbenches.

In other word, this paper presents a non-conventional effort to integrate NURBS models on HBIM open source platform such as FreeCAD, and how they can be parametrized inside the BIM platform.

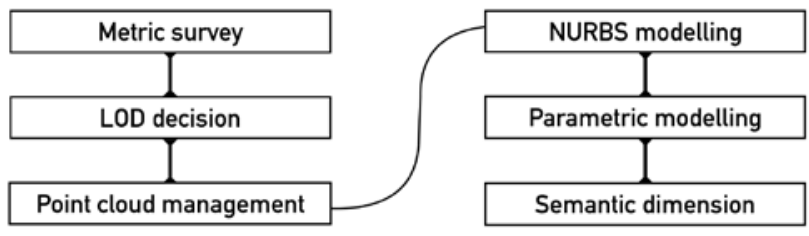

Figure 1. Schema of HBIM creation starting from metric information and NURBS modelling 


\section{METRIC SURVEY}

Cultural Heritage assets, in order to be managed inside an HBIM platform, require to be surveyed with a sufficient level of accuracy by considering the data to be merged and the analysis that the HBIM platform has to allow.

Metric acquisition represents the first step, and it can be carried out by using different techniques, such as LiDAR or photogrammetric approach (e.g. terrestrial, and UAV). The obtained point clouds of the object, with associated RGB values, can be used to create the "as-built" 3D model.

Metric survey is a necessary process of objective knowledge for Cultural Heritage, allowing the registration of complex shapes, surfaces, radiometric information, as well as metric registration, in order to represent, communicate and to help monitoring operations of a surveyed building or site.

Indeed, metric survey includes operations, analysis and of course measurements for the purpose of documentation and awareness increasing of a Cultural Heritage context, for instance structural and constructive features, allowing to detect reality as it is. Managing metric data is a key element to pass to the next step: investigations towards the purpose of creating a model (reality-based model) from a point cloud recorded about a real object.

The rib vault tested for this project has been surveyed by using Faro Focus 3D TSL (terrestrial laser scanner). Resolution of scans has been set up on 1/4 high level and $4 \mathrm{x}$ speed with 122 $\mathrm{kpt} / \mathrm{s}$. After metric data acquisition, the realization of a parametric model for HBIM can be developed. However, Cultural Heritage assets needs a high level of detail as regards geometries and decorations, and for this reason a free form modelling could be a fitting choice. In order to perform this, point clouds management become fundamental.

\section{POINT CLOUD MANAGEMENT}

Point clouds derived from a LiDAR metric survey have to be managed thinking about specific needs and levels of detail that influence the research analysis. In this sense point clouds are the real framework and raw data useful to build reality-based models, NURBS models and parametric models.

Reality-based models, characterized by triangulated meshes and high resolution textures, are generated mostly inside a point cloud management software and they are built operating different creation parameters, depending on levels of detail we need to get, on filtered point clouds.

Despite the possibility of performing different analysis through these high quality reality based models, they still remain anonymous models. Indeed, heavy 3D point cloud does not contribute important information from a semantic point of view, and in this sense, creating a dynamic ontological model could be considered as a good methodology to operate by a multidisciplinary critic analysis (Messaoudi et al, 2018).

In fact, BIM platforms requires dynamic, simplified and lightweight models, because the real big data should be information inside every objects, and this possibility that allows us to know and learn about 3D models.

For this reason is fundamental to manage and simplify point clouds in order to create BIM models.

As far as the example of rib vaults is concerned, point clouds derived from the laser scanner survey has been managed in different ways using 3D Reshaper software. The cleaning phase is fundamental in order to obtain a clear point clouds without noisy elements, as well as for the purpose to create an high quality triangulated mesh, in this case modelled using the two phases option (Figure 2).

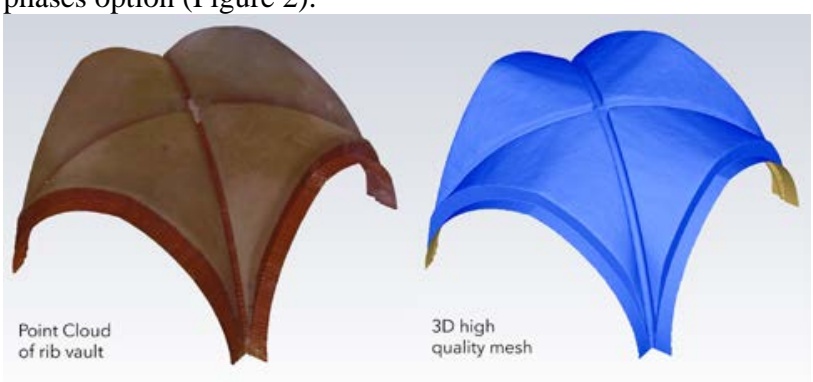

Figure 2. Reality-based model of rib vault.

For the purpose of modelling using NURBS, the rib vault has been sectioned carrying out slices and profiles starting from the high quality point cloud. In fact, by using 3D Reshaper software point clouds has been managed in order to clean and extract planar sections of the vault. In this case sections on $\mathrm{X}, \mathrm{Y}, \mathrm{Z}$ planes have been generated with a regular distance of $10 \mathrm{~cm}$ and a planes thickness of 2,8 mm (Figure 3). The same operation could be carry out also sectioning the triangulated mesh but the precision of these profiles could be affected by the level of detail resulted during mesh generation phase.

Finally, profiles of planar sections of point clouds (Figure 4) have been imported (as CAD element), keeping right coordinate system, in Rhinoceros software.

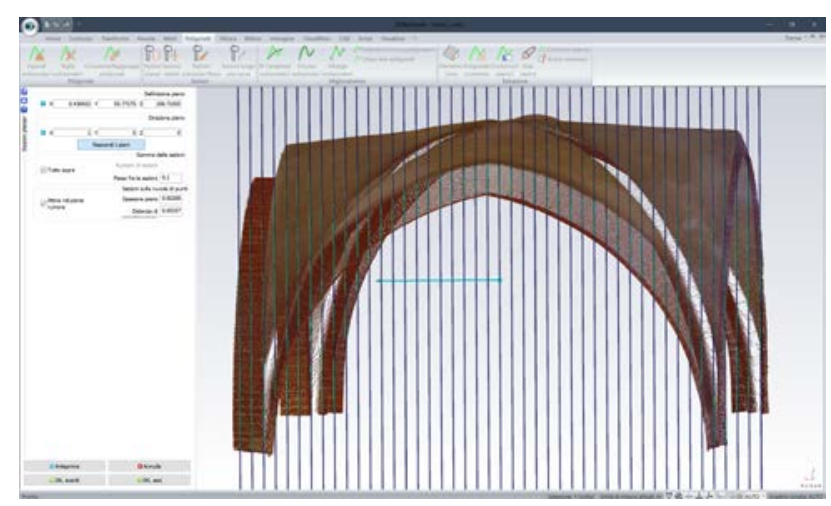

Figure 3. Planar sections on X plane. Distance between planes $10 \mathrm{~cm}$ and a planes thickness of 2,8 $\mathrm{mm}$.

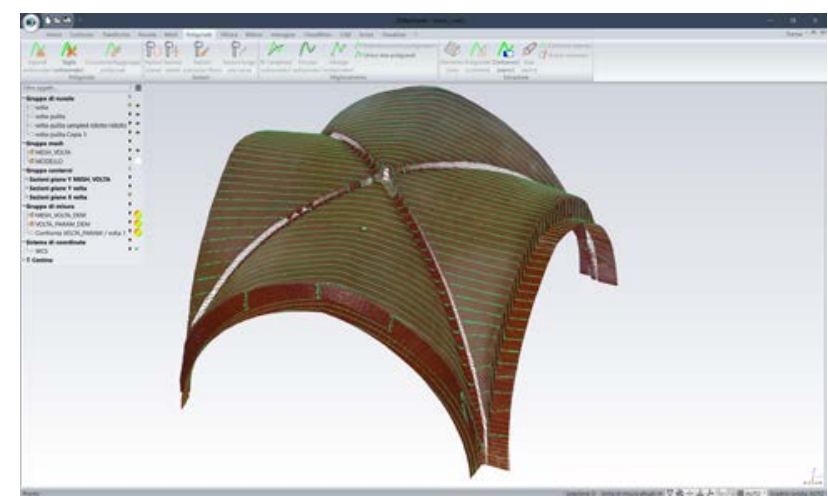

Figure 4. Profile of planar sections on X plane on the point cloud of rib vault. 


\section{3D MODELLING OF COMPLEX ELEMENTS}

Actual commercial BIM modelling software, such as Autodesk Revit or Graphisoft Archicad, allow 3D modelling based on predefined architectural families or very simplified objects, in fact at the moment is very difficult try to realize very complex and high detailed models, for example referred to Cultural Heritage assets (Murphy et al., 2013; Adami et al., 2017).

Indeed, in order to modelling accurately existing historical buildings features the best fitting choice could be guaranteed by a pure 3D modeller such as Rhinoceros. This commercial 3D modeller allow us to create, starting from curves and profile of point clouds, NURBS (Non Uniform Rational Basis-Splines) surfaces by using different command tools (such as loft, sweep, path). Briefly, according with Rhinoceros description, NURBS surfaces or curves are mathematical representation of a threedimensional geometry, which reconstruct any shapes, and for this reason NURBS are a versatile solution in 3D modelling of complexes architectural features (Oreni et al., 2014).

Actually architectural elements could be referred to parametric models usually described inside Architecture Manuals and used by designer in every period of the history: the as-built architectures differ from the designs due to man-workers errors, movements of structures, restorations sometimes not documented, etc. Therefore theoretical models cannot be used to represent the architectural elements to ease the modelling phase. By using free form modelling software, it is possible to generate complex architectural entities directly from point clouds or from slices and profiles.

Despite these advantages, Rhinoceros is not a parametric software and then it not allows to modify the construction parameters associated to a 3D object, even if these operations are possible by editing externally the objects (Tommasi et al, 2016). However, Rhinoceros could be enriched by different tools and plug-in for the purpose to adapt its characteristics to the increasing request of BIM design.

In this sense the plug-in Visual ARQ is a refined software of architectural design that allows adding to Rhinoceros useful tools for the creation of predefined and modern architectural components such as walls, beams, columns, stairs, and so on. In addition, the plug-in guarantee the full compatibility with the IFC standard of BIM objects. Visual ARQ was not designed for existing buildings, especially for historical buildings.

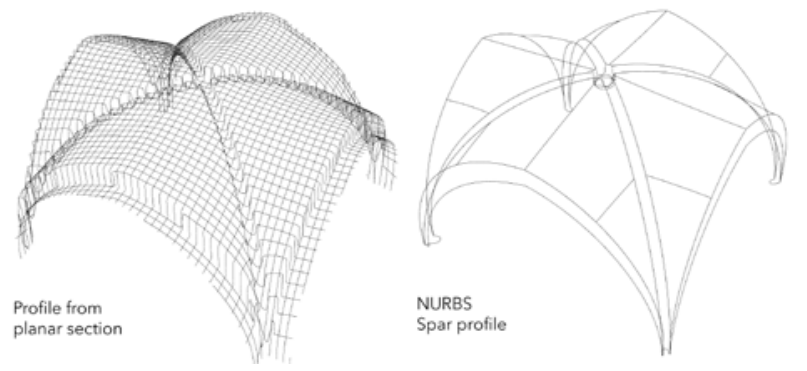

Figure 5. From profiles of planar sections to a custom NURBS spar profile

Established these constraints, the 3D modelling of the rib vault has been carried out by using NURBS geometric and mathematical curves and surfaces, in order to represent all geometries and features. Indeed, starting from planar sections imported, the spar profile has been possible to obtain by drawing NURBS curves directly on sections (Figure 5).

Briefly, as far as the NURBS model of rib vault is concerned, shapes related to the four ribs have been created using sweep command with 2 rails, then for the creation of vaults was used patch command, editing $\mathrm{U}$ and $\mathrm{V}$ subdivision to 50 , and this value is referred especially to the density of curves inside the model. The central intersection between ribs (boss) has been modelled merging patch and loft commands and also using subtractions among surfaces.

Briefly, patch tool fits a surface through selected curves, meshes, point objects, and point clouds; loft tool fits a surface through selected profile curves that define the surface shape; sweep (two rails) tool fits a surface through a series of profile curves that define the surface shape and two curves that define the surface edges (from http://docs.mcneel.com/rhino).

The final model we obtained (Figure 7) has high quality details but it cannot directly be implemented into a BIM platform because it is not a parametric dynamic model. In order to perform this operation it needs to be managed and parametrized.

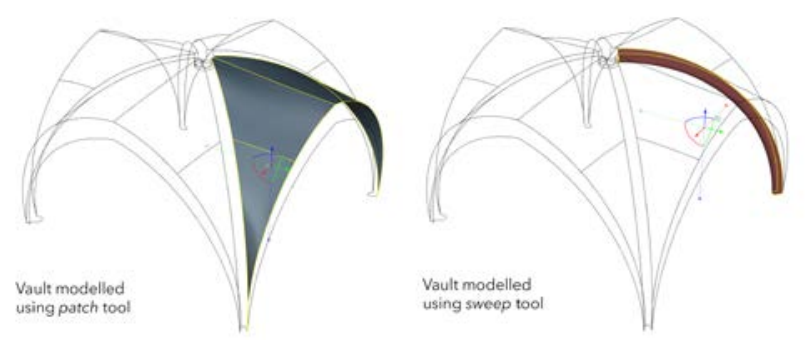

Figure 6. Modelling of the rib vault: patch tool for vaults and sweep tool for ribs

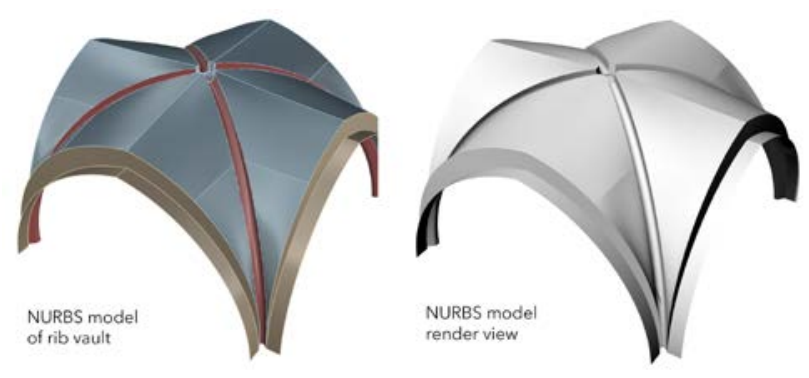

Figure 7. Final NURBS model of the rib vault

\subsection{Model generation and accuracy}

The level of detail (LOD), that represents the scale and the complexity of a model, is a key point to correctly manage the modelling phase, and, in some cases, also the metric survey. Indeed, LOD, which fits the goal of the work (such as quantity and quality of the data to be connected, kind of analysis to be performed, and so on), strongly influences the complexity of the model (Diara et al, 2018). A BIM model, despite it is metrically correct, is not a reality-based model but a critic representation of reality, depending on a specific LOD.

LODs, which are standard for BIM models, are essentially ranging from LOD100 (2D representation) and LOD500 (high quality $3 \mathrm{D}$ representation and all attributes regarding the object).

As regards existing buildings, LOD is referred to the quality of metric survey (in the case of rib vault TSL) and the capacity of modelling software and also of modeller (Fai et al., 2014). However, as recently noticed, the not properly flexibility of BIM protocols, such as databases and modelling techniques, has 
led to introduce a new classification system in order to define not the LOD but the quality of modelling (Banfi F., 2017).

This new system is based on the classification of Grade of Generation (GOG) and Grade of Accuracy (GOA) referred to 3D model.

The workflow here presented, named also scan-to-BIM process, needs different levels of accuracy and generation, especially because, actually, there are no three-dimensional objects corresponding to historical elements inside predefined libraries and families.

In this sense every BIM project, Grade of Generation (GOG) should be referred to the quality of creation and the modelling requirements, while the Grade of Accuracy (GOA) should set out the standard deviation between reality-based point clouds and model, in this case NURBS model (Banfi F., 2017, 2019).

In other words, GOG defines the techniques of modelling and then how the model has been generated. The GOA defines the metric precision between the real object (depending also by the survey accuracy) and the 3D model created manually starting from metric information.

NURBS curves and surfaces are mathematical functions able to interpolate point clouds and slices. As said before, this aspect is fundamental in order to adapt the modelling action to the real object and not the opposite. Indeed, a NURBS model created interpolating point clouds is classified as GOG 10 (maximum level), while a model generated by extracting geometric primitives from slices is referred to GOG 9 (Banfi F., 2019).

This is the case of the rib vault, generated by extracting slices from planar sections, creating a spar profile and modelling directly on it by using sweep, loft, patch functions (GOG 9).

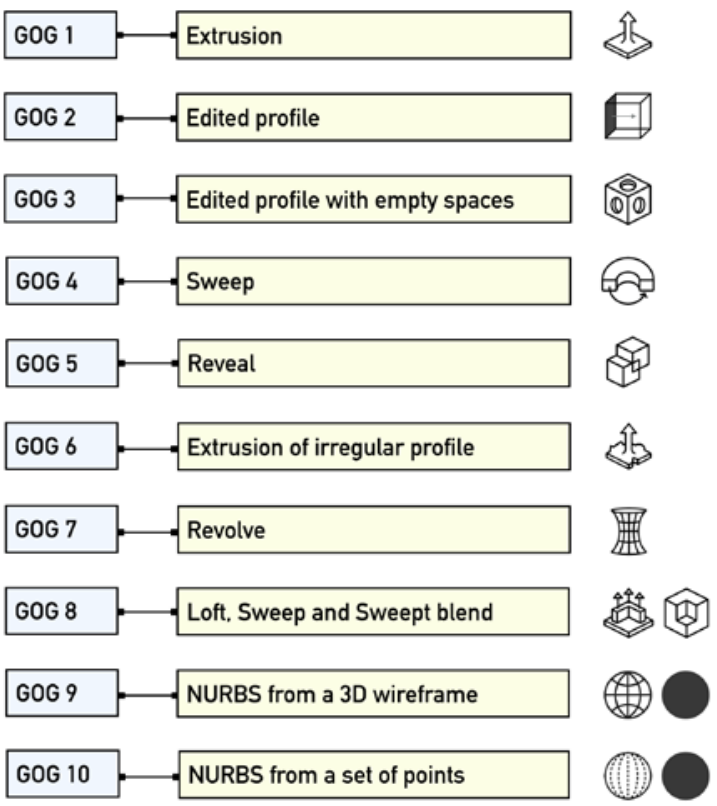

Figure 8. Schematic classification of Grades of Generation (original schema in Banfi F., 2017)

Analysing the rib vault, the GOA is referred to the standard deviation (value that define the average distance between point clouds and the model) that is around $1.7 \mathrm{~cm}$, measured using Cloud Compare software. This value of course is strictly related to the grade of generation of the model (GOG 8 and GOG 9), because NURBS curves and surfaces have been interpolated on profiles generated from planar sections. The interpolation of NURBS directly on point clouds would have generated a smaller deviation (around 5 / $10 \mathrm{~mm}$ ).

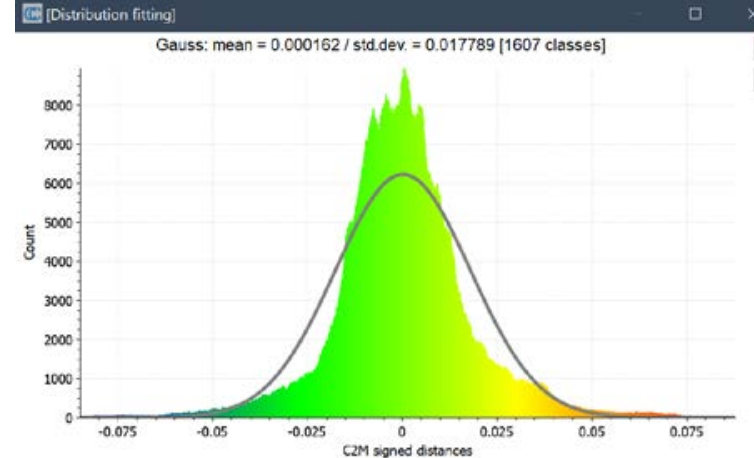

Figure 9. Graphic of standard deviation between point clouds and the model of rib vault

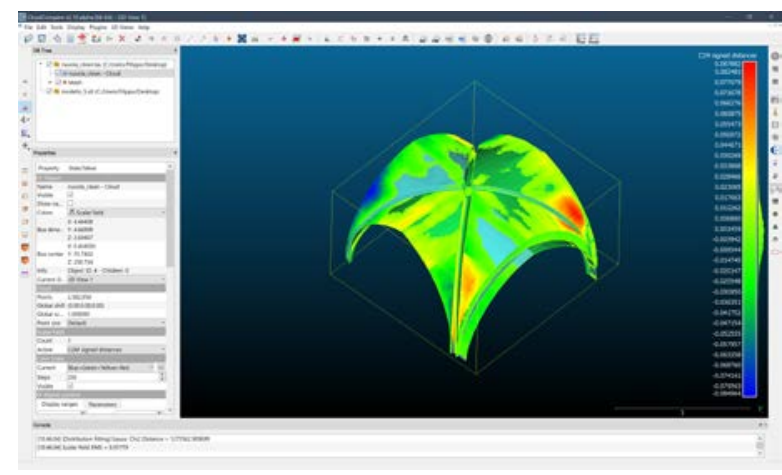

Figure 10. Distance between point clouds and the model of rib vault (overlapped)

\section{NON-CONVENTIONAL HBIM PLATFORM}

Despite these limitations, it has been proved the integration between BIM commercial application and NURBS modelling, in order to enrich the model creation phase, and then the generative process itself (Oreni et al., 2014; Banfi F., 2016, 2017).

This interesting integration could be tested also using different solutions. Indeed, this experimentation has been carried out especially for the purpose of integrating free form models into a non-conventional open source BIM platform, as FreeCAD software. (Tested at latest stable version 0.18).

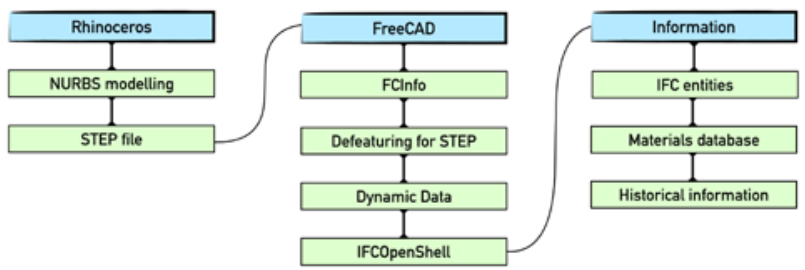

Figure 11. Schematic workflow from NURBS modelling to open source HBIM

\subsection{FreeCAD as BIM platform}

FreeCAD is an open source parametric CAD/BIM software based on the presence of different workbenches. Being a 3D parametric modeller, the objects that can be generated have geometries and shapes that depend on their properties.

This software is almost bare from a lot of necessary tools and it 
requires to be compiled with libraries, packages and plug-in easily connected inside the software.

The use and customization of features require different packages and tools, such as Phyton Installer, PIP (package manager for python), CMake (an open-source, cross-platform family of tools designed to build, test and package software), Homebrew package and XCode (Diara et al., 2018).

For the purpose of developing an HBIM platform BIM, Arch, Draft, Part and Part Design workbenches are probably the most important modules that could be used in order to design and create parametric objects.

Indeed, Arch and BIM workbenches have many tools to define the site and building of a project, and of course the architectural elements of a building, such as wall, structure, and component. Anyway, all these features are thought for architectural design and new constructions (Diara et al., 2018).

\subsection{From NURBS to HBIM}

NURBS surfaces of the rib vault created using Rhinoceros software have been exported in STEP format (Standard for the Exchange of Product model data), defined by ISO normative 10303-21. By using STEP file, the information related to the object are recognize by different coding protocols and could be sharing in a three-dimensional model.

For the purpose of parametrize NURBS surfaces exported using STEP files, FreeCAD could be implemented by useful custommade tools developed by the big community of FreCAD, such as: Defeaturing workbench, FCInfo, Dynamic Data. Defeaturing workbench is an add-on thought for editing STEP models, removing of the selected features from the model. Using this add-on it is also possible to direct model the object, also when the history of operations is unavailable, and this is the case for 3D STEP objects. Defeaturing can also useful to remove proprietary details of the model before sharing it. Finally, this add-on allows converting a 3D STEP object into a parametric solid. However, this operation is possible by extruding STEP object using extrusion command tool, in part workbench, which allows to create a parametric solid starting from shapes and surfaces.

The FCInfo is a simple macro add-on that gives a series of information (coordinates, length, area, volume, weight, etc...) about the selected surface or shape and can display all the characteristics of objects not directly created on FreeCAD, because the default data viewer of the software show basic information of imported objects and not entire features.

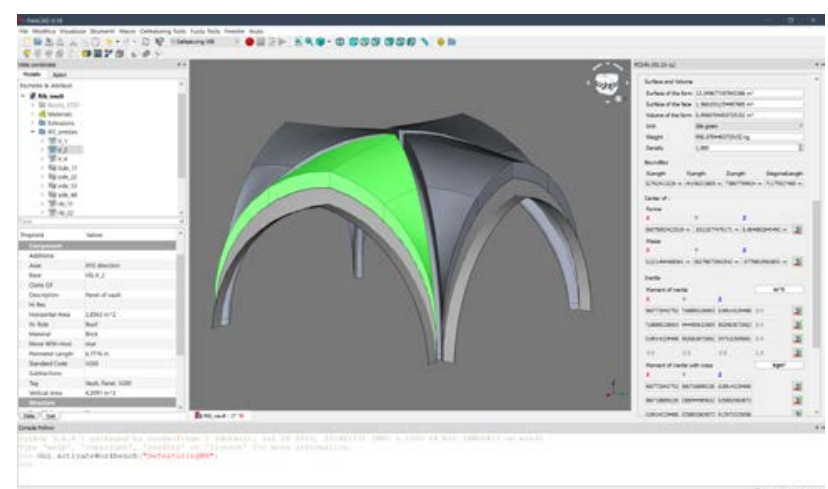

Figure 12. FreeCAD platform and creation of parametric object by using arch, defeaturing workbenches and dynamic data

The Dynamic Data workbench allows creating custom FeaturePython objects to serve as containers for customizable properties. These properties can then be used in the same way as cells in a CSV database in order to collect and modify, in real time, the parameters. Moreover, files containing these Dynamic Data objects can be shared with other users who do not have this workbench installed on there systems and yet will still remain fully functional.

By using these tools the parametrization of rib vault has been conducted: STEP file of rib vault has been edited using Defeauturing tools in order to editing every shape related to the vault. Moreover, the FreeCAD extrusion command allows to obtain parametric objects in addition to the dynamic data tools, which have helped the inclusion and customization of specific parameters on the platform. Through the dynamic data tools flexible parameters have been included into objects, and the modification of these custom properties involves modification also for the object. As far as the extrados of the rib vault, it was modelled extruding NURBS surfaces by measuring and calculating the dimension of bricks (around 26-28 cm including the plaster).

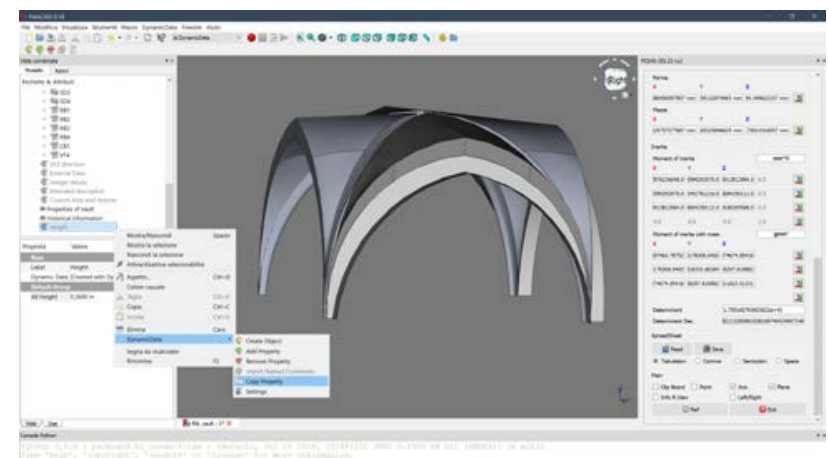

Figure 13. Detail of dynamic data tools inside FreeCAD

\subsection{Semantic dimension}

In addition to the previous tools, IfcOpenShell is a fundamental open source library of FreeCAD that allows managing IFC format, necessary requirement for a BIM environment. Indeed, IFC (Industry Foundation Classes) open format, developed by "buildingSMART" is referred to a relational object characterized by different patterns and layers that includes different classes.

Indeed, beyond geometry, elements and surfaces extracted from point clouds must be assigned to classes of objects and typological sub divisions (Macher et al, 2017): in this sense an extruded surface derived from point clouds become for example a parametric wall (based on real volumes) with specific properties and materials. This kind of entities can be defined inside IFC standard, which could be considered a dynamic custom-made database: in fact, it is possible to include a lot of information, such as the object's material, the physical relationships with other models, architectural roles (Diara et al., 2018).

For this reason, IFC objects inside HBIM platform assume a kind of semantic dimension, because are characterized by thematic descriptions such as architectural entities, roles, material, historical information (Messaoudi et al, 2018) that should be managed inside relational DBMS (database management system). DBMS structures allow collecting and querying data that can be used to design and manage future interventions in a specific context.

As far as the rib vault is concerned, the IfcOpenShell library enabled the implementation of architectural entities inside parametric objects. However, the entity vault is not included on 
the default IFC schema of FreeCAD, even if the roles and description parameters are customizable (Diara et al., 2018). In this case, entities like Roof and BuildingElement could be used, even if they are not properly suitable for a complex architectural element as a rib vault. Indeed, IFC definitions are thought for architecture design and technical installations and for this reason they are not exactly fitting for Cultural Heritage assets.

The information regarding the rib vault has been stored also into CSV database inside FreeCAD, which communicates with dynamic data parameters and other kind of information (for example historical) as well.

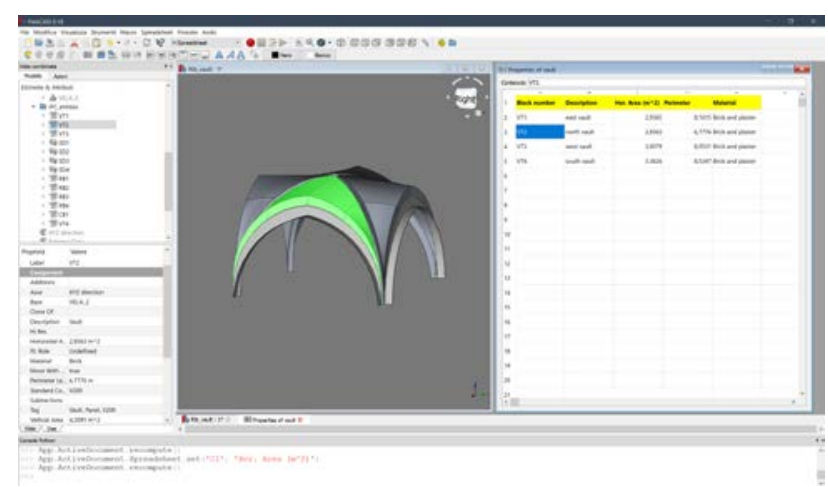

Figure 14. CSV spreadsheet referred to the rib vault properties inside FreeCAD

The creation of a relational database is a key point for an HBIM project for the purpose of collecting and querying geometric and alphanumeric data that can be used to design and manage future interventions, to document the present situation, or recreate a previous situation of the object (Lo Turco et al, 2017). In this sense database become fundamental and the principal aims of HBIM projects should be the collection, management, and publication of large amount of information (structural situation, materials, historical phases, etc.) that are becoming crucial features into BIM platforms (Brusaporci et al, 2018).

\section{CONCLUSIONS}

Manual free form modelling is significant because historical architectural elements must be interpreted and approximated combining the imperfection of the real geometry and the process of modelling shapes from the initial point cloud and due to the chosen level of detail (Lo Turco et al, 2017).

In this sense, parametric models derived from NURBS modelling, could be thought as a kind of anastylosis for shape affinity, considering it as a digital dynamic reconstruction of historic building using elementary shapes (primitives), observing right geometries and volumes.

As far as the tested software are concerned, the open source software FreeCAD could provide to adapt its features and tools for the purpose of representing, defining and classifying Cultural Heritage assets, in fact by using this software it is possible to define architectural entities and geometric relationships between elements, (e.g. according to the real situation of the architectural composition). Anyway, due to its objective limitations and unstable tools, FreeCAD could combine its work with other 3D modelling software, such as Rhinoceros, that also includes IFC interoperability. This kind of cooperation between different software (especially in a conceptual point of view) could be a suitable solution when we run into critical point such as on a particular modelling or entities classifications.

Indeed, Cultural Heritage assets must be supported by custom simplified levels that have to be analysed as well as possible for the purpose to represent anomalies in the geometries. (Georgopoulos et al., 2013).

However, there are advantages and drawbacks to the utilization of open source and commercial software. Software platforms, like FreeCAD are developed to have a dynamic and full customizable CAD/BIM software, despite the presence of few tools and the minimal of required computer science skill. Commercial software, as Rhinoceros, are thought for a massive diffusion in the 3D modelling area; its tools could be increased by add-ons or plug-in. Rhinoceros is a complete 3D modeller, not a BIM software, and it has high cost and does not allow the full managing and customization of IFC objects for Cultural Heritage assets.

In addition to this, carrying out an HBIM project testing different software we could have a lot of file with any format and extension. The management of an HBIM model involves also to work inside many software and OS platforms and it could be dangerous for file compatibility and then for the project stability in general. Thus, should be necessary planning in details the entire project, including software and OS we could use, in order to ensure the right sharing possibilities.

Moreover, another important aspect of sharing process regarding the portability data and its information. In this sense mobile devices plays a fundamental role (Barazzetti et al., 2015). Indeed, actual mobile applications have enriched the panorama of possibility for the purpose to exchange threedimensional data in a smart and easy way.

\section{ACKNOWLEDGMENTS}

This work was supported and funded by the GAMHer project (Geomatics data Acquisition and Management for landscape and built Heritage in a European perspective), a 3 years project financed under the Italian PRIN 2015 framework (Progetti di Ricerca di Rilevante Interesse Nazionale).

\section{REFERENCES}

Adami, A., Scala, B., and Spezzoni, A., 2017. Modelling and accuracy in a BIM environment for planned conservation: the apartment of Troia of Giulio Romano. In: The International Archives of the Photogrammetry, Remote Sensing and Spatial Information Sciences, XLII-2/W3, 17-23, 2017.

Banfi F., 2016. Building Information Modelling - A Novel Parametric Modeling Approach Based on 3D Surveys of Historic Architecture. Digital Heritage. Progress in Cultural Heritage: Documentation, Preservation, and Protection. pp.112. 2016

Banfi F., 2017. BIM orientation: grades of generation and information for different type of analysis and management process. In: The International Archives of the Photogrammetry, Remote Sensing and Spatial Information Sciences, XLII-2/W5, pp. 57-64

Banfi, F., 2019. The integration of a scan-to-HBIM process in BIM application: the development of an add-in to guide users in Autodesk Revit. In: The International Archives of the Photogrammetry, Remote Sensing and Spatial Information Sciences, XLII-2/W11, 141-148, 2019. 
Barazzetti, L., 2016. Parametric as-built model generation of complex shapes from point clouds. In: Advanced Engineering Informatics, 30 (2016) 298-311, 2016.

Barazzetti L., Banfi F., Brumana R., Oreni D., Previtali M., Roncoroni F., 2015. HBIM and augmented information: towards a wider user community of image and range-based reconstructions, In: The International Archives of the Photogrammetry, Remote Sensing and Spatial Information Sciences, XL-5/W7, 35-42, 2015

Beltramo, S., Diara, F., and Rinaudo, F., 2019. Evaluation of an integrative approach between HBIM and architecture history. In: The International Archives of the Photogrammetry, Remote Sensing and Spatial Information Sciences, XLII-2/W11, 225229, 2019.

Brusaporci, S., Maiezza, P., Tata, A., 2018. A framework for architectural heritage HBIM semantization and development. In: The International Archives of the Photogrammetry, Remote Sensing and Spatial Information Sciences, Volume XLII-2, pp.179-184, 2018.

Diara, F., Rinaudo, F., 2018. Open source HBIM for Cultural Heritage: a project proposal. In: The International Archives of the Photogrammetry, Remote Sensing and Spatial Information Sciences,Vol. XLII-2, 2018 ISPRS TC II pp.303-309, 2018.

Fai, S., Rafeiro, J., 2014. Establishing an appropriate level of detail (LoD) for a building information model (BIM) - West Block, Parliament Hill, Ottawa, Canada. ISPRS Annals of the Photogrammetry, Remote Sensing and Spatial Information Sciences, 2(5), pp.123-130

Georgopoulos A., Brumana R., Oreni D., Raimondi A., Barazzetti L., Bregianni A., 2013. HBIM for Documentation, Dissemination and Management of Built Heritage. The Case Study of St. Maria in Scaria d'Intelvi. International Journal of Heritage in the Digital Era. 2. 10.1260/2047-4970.2.3.433.
Lo Turco, M., Mattone, M., Rinaudo, F., 2017. Metric survey and BIM technologies to record decay conditions. In: The International Archives of the Photogrammetry, Remote Sensing and Spatial Information Sciences.Vol. XLII-5/W1, pp.261-268

Logothetis, S., Karachaliou, E., Stylianidis, E., 2017. From OSS $\mathrm{CAD}$ to BIM for Cultural Heritage digital representation. In: The International Archives of the Photogrammetry, Remote Sensing and Spatial Information Sciences. Vol. XLII- 2/W3, 2017.

Macher, H., Landes, T., Grussenmeyer, P., 2017. From Point Clouds to Building Information Models: 3D Semi-Automatic Reconstruction of Indoors of Existing Buildings. In: Applied Sciences 2017, 7, 1030.

Messaoudi, T., Véron, P., Halin, G., De Luca, L., 2018. An ontological model for the reality-based 3D annotation of heritage building conservation state. In: Journal of Cultural Heritage. Vol. 29, pp.100-112. 2018

Murphy, M., McGovern, E., Pavia, S., 2013. Historic Building Information Modelling - Adding intelligence to laser and image based surveys of European classical architecture. ISPRS Journal of Photogrammetry and Remote Sensing, 76, 89-102.

Oreni D., Brumana R., Banfi F., Bertola L., Barazzetti L., Cuca B., Previtali M., Roncoroni F., 2014. Beyond Crude 3D Models: From Point Clouds to Historical Building Information Modeling via NURBS. Lecture Notes in Computer Science. 8740. 166-175. 10.1007/978-3-319-13695-0_16.

Tommasi, C., Achille, C., and Fassi, F., 2016. From point cloud to BIM: a modelling challenge in the cultural heritage field. In: The International Archives of the Photogrammetry, Remote Sensing and Spatial Information Sciences, XLI-B5, 429-436. 2016 